\title{
Effects of Vasculogenic Mimicry on Postoperative Recurrence and Progression of Non-Small Cell Lung Cancer
}

\section{Baoyong Ling1, Aiqin Peng², Jijun You'3 ${ }^{3}$ Zhisheng Zhang1, Weichun Li', Yayun Qian', Yanqing Liu5, Jue} Chen $1,5,6 *$

1Department of Oncology, Yangzhou University affiliated the Second People's Hospital of Taizhou, Taizhou, Jiangsu Province, China

2Department of Nursing, Yangzhou University affiliated Wutaishan Hospital, Yangzhou, Jiangsu Province, China

${ }^{3}$ Department of Orthopedic, Yangzhou University affiliated the Second People's Hospital of Taizhou, Taizhou, Jiangsu Province, China

${ }^{4}$ Department of Respiratory, Taizhou Jiangyan Traditional Chinese Medicine Hospital, Taizhou, Jiangsu Province, China

${ }^{5}$ Institute of Medicine, Yangzhou University, Yangzhou, Jiangsu Province, China

${ }^{6}$ Department of Oncology, Yangzhou University Affiliated Hospital, Yangzhou 225500, Jiangsu Province, China

*Corresponding author: Jue Chen, 1019924551@qq.com

\begin{abstract}
Vasculogenic mimicry (VM) in lung cancer shortens overall survival (OS) but its' associations with postoperative recurrence and progression of early non-small cell lung cancer (NSCLC) remain unclear. The purpose of this study was to analyze the association of VM with postoperative recurrence and progression of NSCLC as well as the effect of VM on postoperative recurrence-free survival (RFS). This study included NSCLC patients and detected VM in surgical specimens. The associations of VM with the recurrence and progression were analyzed to assess the effect of VM on postoperative RFS in NSCLC. A total of 80 NSCLC cases were followed up for 3 years. During follow-up, 35 cases showed recurrence and progression where $5(6.25 \%)$ cases had simple local recurrence and the other $30(37.5 \%)$ cases had distant metastasis. The recurrence and progression rates in the first, second, and third years were $12.50 \%, 23.75 \%$, and $7.50 \%$, respectively. The median RFS was 14.2 months. VM was detected in 30 out of 80 cases and was significantly correlated with tumor differentiation $(r=0.365)$ and clinical stage $(r=0.374)$ (both, $P=0.001)$. Local recurrence of NSCLC was not correlated with $\mathrm{VM}$, unlike distant metastasis $(\mathrm{r}=0.598, \mathrm{P}<0.001)$. Average RFS was significantly longer in NSCLC patients without VM compared with the VM group 3 years post-operation $(32$ months versus 18 months, log-rank test $\mathrm{P}<0.001$ ). Considering these, VM is significantly correlated with postoperative distant metastasis of NSCLC in which it is of a certain value for predicting poor prognosis in NSCLC.
\end{abstract}

Keywords: Non-small cell lung cancer; Vasculogenic mimicry; Recurrence and progression; Recurrence-free survival

Publication date: July 2021; Online publication: July 30, 2021

\section{Introduction}

Lung cancer is currently the most common malignant tumor with the highest morbidity and mortality rates worldwide and about $80-85 \%$ of first-time diagnosed cases each year being NSCLC ${ }^{[1]}$. At present, surgical resection, guided by TNM staging is the main treatment for pre-stage IIIA patients. However, due to the heterogeneity of lung cancer, despite complete surgical resection and postoperative adjuvant therapy, nearly 
$80 \%$ develop distant metastasis ${ }^{[2]}$. Screening of high-risk factors related to postoperative progression of NSCLC has an important clinical significance for detecting NSCLC patients who are prone for progression.

The overall postoperative survival of VM-positive NSCLC patients is significantly shortened. ${ }^{[2]}$ Epithelial to mesenchymal transition (EMT) and stem-like differentiation of tumor cells constitute the key events for VM formation in which they are relevant promoters of tumor metastasis ${ }^{[3]}$. In formed VM, tumor cells are in direct contact with blood circulation and they can easily break off into the peripheral circulation. Therefore, the formation mechanism and structural characteristics of VM are extremely beneficial to the hematogenous spread of malignant tumors ${ }^{[4]}$. There are a few reports on the associations of VM with postoperative recurrence and progression of NSCLC and their possible mechanisms.

It is hypothesized that VM promotes postoperative distant recurrence and progression of NSCLC, hence the risk of postoperative distant recurrence and progression can be determined by assessing the presence of VM in surgical specimens of NSCLC cases. For this purpose, this study was designed to assess the value of VM in determining NSCLC recurrence and metastasis. The findings from this study would provide a reference for further improvement in the postoperative prognostic evaluation of NSCLC.

\section{Material and methods}

\subsection{Enrolment of NSCLC cases}

This study was a single-center, cross-sectional study. Cases were enrolled from March 1, 2015 to February 29, 2016 and the follow-up period was for 3 years. NSCLC tissue samples were collected from the Clinicopathology Center in Taizhou Second People's Hospital, affiliated to Yangzhou University. The diagnostic and staging criteria were based on the National Comprehensive Cancer Network (NCCN) Guidelines version $2014^{[5]}$, preoperative computed tomography (CT), magnetic resonance imaging (MRI), or positron emission tomography (PET)-CT imaging. The diagnosis of NSCLC was confirmed by pathologists through microscopy after surgery and those cases with very low differentiation that could not be confirmed as NSCLC were identified by immunohistochemical staining. The exclusion criteria were pulmonary metastatic tumors, preoperative antineoplastic therapy (neoadjuvant chemoradiotherapy, immunotherapy and targeted therapy, or antineoplastic therapy with traditional Chinese medicine), perioperative mortality due to severe complications, estimated postoperative survival less than 3 months, postoperative stage more than IIIB, postoperative immunosuppressive therapy for underlying diseases more than 1 month, age $>90$ years or < 18 years, or cases with T4 and N2-3 in the TNM staging (preoperative neoadjuvant therapy). The demographic and pathological data were collected from the electronic medical record system in Taizhou Second People's Hospital. The research protocol was approved by the Ethics Committee of Taizhou Second People's Hospital (No. TZEYLL 20150107). All selected cases or their legal representatives had provided signed informed consent.

\subsection{Postoperative follow-up}

The patients were followed up every month in the first year, every 3 months in the second, and every 3-6 months in the third. Follow-ups were conducted in the form of outpatient care or hospitalizations. Followup examinations included CT, MRI, and PET-CT. For new lesions of highly suspected metastasis that could not be confirmed by imaging, a second puncture biopsy was performed for confirmation. RFS was defined as the time from the date of surgery to recurrence, progression, death, or the end of the study. 


\subsection{Detection and identification of $\mathrm{VM}$}

VM was detected by CD34-PAS (periodic acid-Schiff) double staining using paraffin-embedded tissue samples according to a previous method ${ }^{[6]}$. Typical vascular structure under microscope and CD34+ was identified as endothelial dependent vessel (EDV) while CD34-/PAS+ vascular structure was considered as VM.

\subsection{Statistical methods}

Statistical Package for the Social Sciences (SPSS) version 19.0 (SPSS Inc., Chicago, IL) was used to analyze the data. Fisher's exact test was used for categorical variables. Spearman correlation was performed to assess the correlation between two categorical variables. Kaplan-Meier analysis was carried out to evaluate clinical and pathological characteristics that might affect RFS while log-rank test was used to assess the effects of single factors on RFS. The Cox regression model (COX) was used for multivariate analysis of prognostic factors. Two-sided $\mathrm{P}<0.05$ was considered as statistically significant.

\section{Results}

\subsection{Baseline characteristics}

A total of 80 NSCLC surgical cases were included. The average age of the patients that were included in this study was $65.03 \pm 8.75$ years old (38-85 years old). There were 57 male patients $(71.25 \%)$ and 29 smokers (36.25\%). Among the 80 cases of NSCLC, adenocarcinoma was slightly more represented than squamous cell carcinoma (43 versus 37). The proportion of patients with postoperative stage I-II was $83.75 \%$. The patients' baseline features are shown in Table 1.

Table 1. Demographic and clinical characteristics of NSCLC patients

\begin{tabular}{ccc}
\hline Baseline characteristics & All cases $(\mathrm{n}=80)$ & Number of cases $(\%)$ \\
\hline Age & $<65$ years old & $33(41.25 \%)$ \\
& $\geq 65$ years old & $47(58.75 \%)$ \\
Gender & Male & $57(71.25 \%)$ \\
& Female & $23(28.75 \%)$ \\
History of smoking & Yes & $29(36.25 \%)$ \\
& No & $51(63.75 \%)$ \\
Pathological type & Squamous carcinoma & $37(46.25 \%)$ \\
Differentiation degree & Adenocarcinoma & $43(53.75 \%)$ \\
& High differentiation & $12(15.00 \%)$ \\
T stage & Moderate differentiation & $49(61.25 \%)$ \\
& Low differentiation & $19(23.75 \%)$ \\
N stage & T1 & $18(22.50 \%)$ \\
& T2 & $39(48.75 \%)$ \\
Clinical stage & T3 & $23(28.75 \%)$ \\
& N0 & $36(45.00 \%)$ \\
& N1 & $44(55.00 \%)$ \\
& Stage I & $26(32.50 \%)$ \\
& Stage II & $41(51.25 \%)$ \\
& Stage III & $13(16.25 \%)$ \\
\hline
\end{tabular}




\subsection{Distribution and structural characteristics of VM in NSCLC}

A total of $30(37.50 \%$; 30/80) NSCLC specimens had VM structures where $12(32.43 \%$; 12/37) cases involved squamous carcinomas and $18(41.86 \%$; 18/43) involved adenocarcinomas. There was no significant difference in the detection of VM between the adenocarcinoma and squamous cell carcinoma groups $(\mathrm{P}=0.385)$. The lumen type was predominant in squamous carcinoma cases while the lumen and matrix types were common in adenocarcinoma cases (Figure 1). The lower the differentiation, the more likely VM occurred $(\mathrm{r}=0.365, \mathrm{P}=0.001)$. The later the postoperative clinical stage, the higher the probability of VM occurrence $(\mathrm{r}=0.374, \mathrm{P}=0.001)$ (Table 2).

A

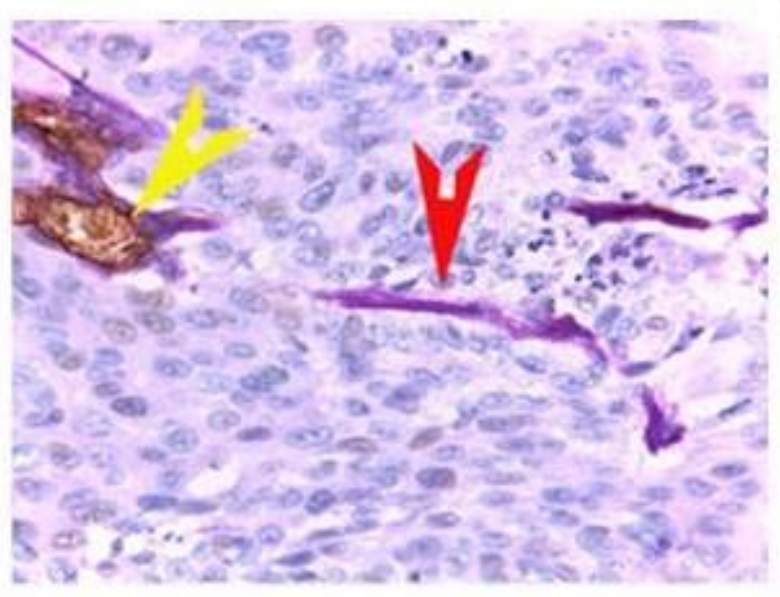

C

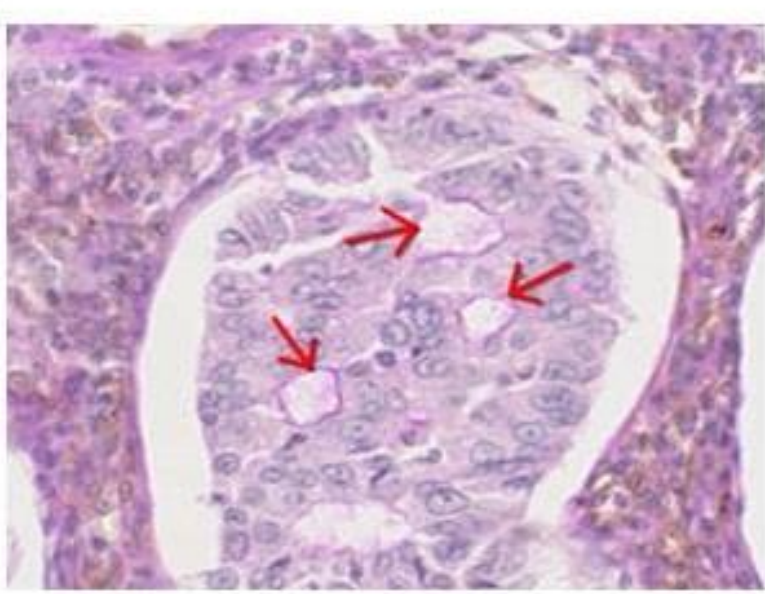

B

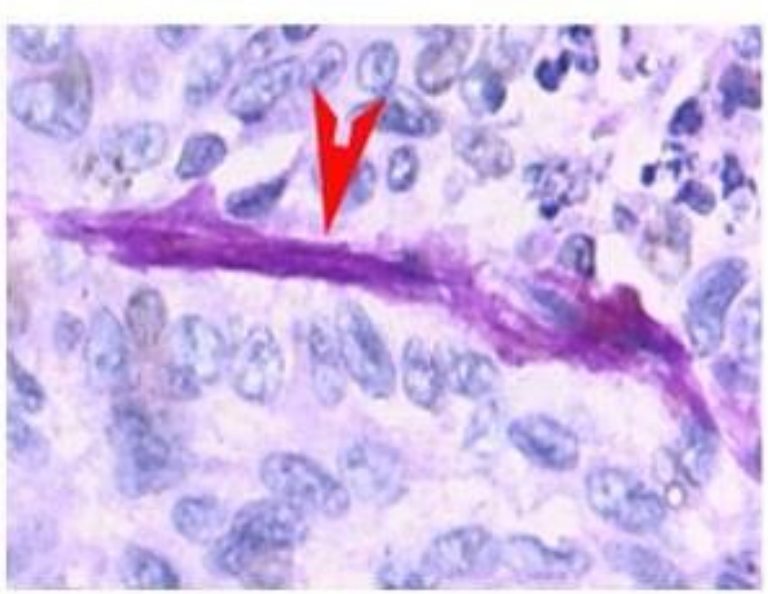

D

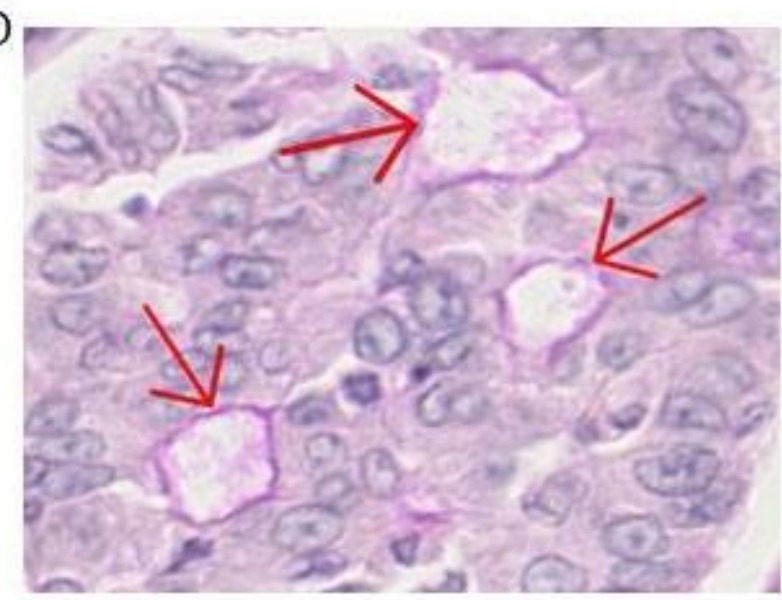

Figure 1. Occurrence of VM in NSCLC. A and B: Morphologies of VM in squamous carcinoma (yellow arrow: EDV; red arrow: VM); C and D: Morphologies of VM and EDV in adenocarcinoma. 
Table 2. Associations of baseline characteristics with VM

\begin{tabular}{|c|c|c|c|}
\hline Variable & $\mathrm{VM}+(\mathrm{n}=30)$ & Correlation coefficient & $\mathrm{P}$ value \\
\hline Age & & 0.033 & 0.773 \\
\hline$<65$ years old & 13 & & \\
\hline$\geq 65$ years old & 17 & & \\
\hline Gender & & 0.021 & 0.851 \\
\hline Male & 21 & & \\
\hline Female & 9 & & \\
\hline History of smoking & & 0.047 & 0.308 \\
\hline Yes & 10 & & \\
\hline No & 20 & & \\
\hline Pathological type & & 0.097 & 0.392 \\
\hline Squamous carcinoma & 12 & & \\
\hline Adenocarcinoma & 18 & & \\
\hline Differentiation degree & & 0.365 & 0.001 \\
\hline High differentiation & 4 & & \\
\hline Moderate differentiation & 11 & & \\
\hline Low differentiation & 15 & & \\
\hline T stage & & 0.412 & $<0.001$ \\
\hline $\mathrm{T} 1$ & 1 & & \\
\hline $\mathrm{T} 2$ & 14 & & \\
\hline $\mathrm{T} 3$ & 15 & & \\
\hline $\mathrm{N}$ stage & & 0.389 & $<0.001$ \\
\hline No & 6 & & \\
\hline $\mathrm{N} 1$ & 24 & & \\
\hline Clinical stage & & 0.374 & 0.001 \\
\hline I & 4 & & \\
\hline II & 17 & & \\
\hline III & 9 & & \\
\hline
\end{tabular}

\subsection{Postoperative recurrence and progression of NSCLC}

Until April 10, 2019, a total of 78 cases that were included in the initial analysis had completed the postoperative follow-up for 3 years (follow-up rate of 97.5\%). A total of 2 cases were lost to follow-up where one of them withdrew in May 2017 and another, in November 2018. The lost cases were considered to be progressive. The median follow-up duration was 21.2 months (6.4-36 months). During the follow-up period, 35 cases $(43.75 \%$; 35/80) were confirmed with recurrence and/or distant metastasis and a median RFS of 14.2 months $(5.2-25.9$ months) was obtained. A total of 10 cases $(12.50 \% ; 10 / 80)$ in the first year, $19(23.75 \%$; 19/80) in the second year, and $6(7.50 \% ; 6 / 80)$ in the third year showed postoperative recurrence (Table 3). Out of the 35 patients with recurrence, $5(6.25 \%$; 5/80) of them had simple local recurrence while the other $30(37.50 \% ; 30 / 80)$ had distant metastasis. The distant metastatic cases included 9 oligometastatic cases $(30 \% ; 9 / 30)$ and 21 multiple metastatic cases $(70 \% ; 21 / 30)$. The most common metastatic site was the lung $(60.00 \% ; 21 / 35)$, followed by the brain $(42.86 \% ; 15 / 35)$, liver $(31.43 \% ; 11 / 35)$, bone $(22.86 \%$; 8/35), and adrenal gland (14.29\%; 5/35) (Figure 2). Meanwhile, a case of squamous carcinoma of the lung with distant metastases developed skin metastasis over the left shoulder. 
Table 3. Recurrence and progression of postoperative NSCLC

\begin{tabular}{|c|c|c|c|c|c|}
\hline Variable & $\begin{array}{l}\text { Progression } \\
(\mathrm{n}=35, \%)\end{array}$ & $\begin{array}{l}\text { First year } \\
(\mathrm{n}=10, \%)\end{array}$ & $\begin{array}{r}\text { Second year } \\
(\mathrm{n}=19, \%)\end{array}$ & $\begin{array}{l}\text { Third year } \\
(\mathrm{n}=6, \%)\end{array}$ & $\mathrm{P}$ value \\
\hline Age & & & & & 0.012 \\
\hline$<65$ years old & $9(27.27 \%)$ & 4 & 4 & 1 & \\
\hline$\geq 65$ years old & $26(55.32 \%)$ & 6 & 15 & 5 & \\
\hline Gender & & & & & 0.131 \\
\hline Male & $28(49.12 \%)$ & 7 & 15 & 6 & \\
\hline Female & $7(30.43 \%)$ & 3 & 4 & 0 & \\
\hline History of smoking & & & & & 0.284 \\
\hline Yes & $15(51.72 \%)$ & 4 & 8 & 3 & \\
\hline No & $20(39.22 \%)$ & 6 & 11 & 3 & \\
\hline Pathological type & & & & & 0.019 \\
\hline Squamous carcinoma & $11(29.73 \%)$ & 3 & 8 & 0 & \\
\hline Adenocarcinoma & $24(55.81 \%)$ & 7 & 11 & 6 & \\
\hline Differentiation degree & & & & & 0.007 \\
\hline High differentiation & $6(50.00 \%)$ & 1 & 5 & 0 & \\
\hline Moderate differentiation & $13(26.53 \%)$ & 6 & 5 & 2 & \\
\hline Low differentiation & $16(84.21 \%)$ & 3 & 9 & 4 & \\
\hline T stage & & & & & $<0.001$ \\
\hline $\mathrm{T} 1$ & 0 & 0 & 0 & 0 & \\
\hline $\mathrm{T} 2$ & $14(35.90 \%)$ & 1 & 10 & 3 & \\
\hline $\mathrm{T} 3$ & $21(91.30 \%)$ & 9 & 9 & 3 & \\
\hline $\mathrm{N}$ stage & & & & & $<0.001$ \\
\hline No & $1(2.78 \%)$ & 0 & 1 & 0 & \\
\hline $\mathrm{N} 1$ & $34(77.27 \%)$ & 10 & 18 & 6 & \\
\hline Clinical stage & & & & & $<0.001$ \\
\hline I & 1 & 0 & 1 & 0 & \\
\hline II & $21(51.22 \%)$ & 5 & 10 & 6 & \\
\hline III & $13(100 \%)$ & 5 & 8 & 0 & \\
\hline VM & & & & & $<0.001$ \\
\hline No & $10(20.00 \%)$ & 2 & 6 & 2 & \\
\hline Yes & $25(83.33 \%)$ & 8 & 13 & 4 & \\
\hline
\end{tabular}




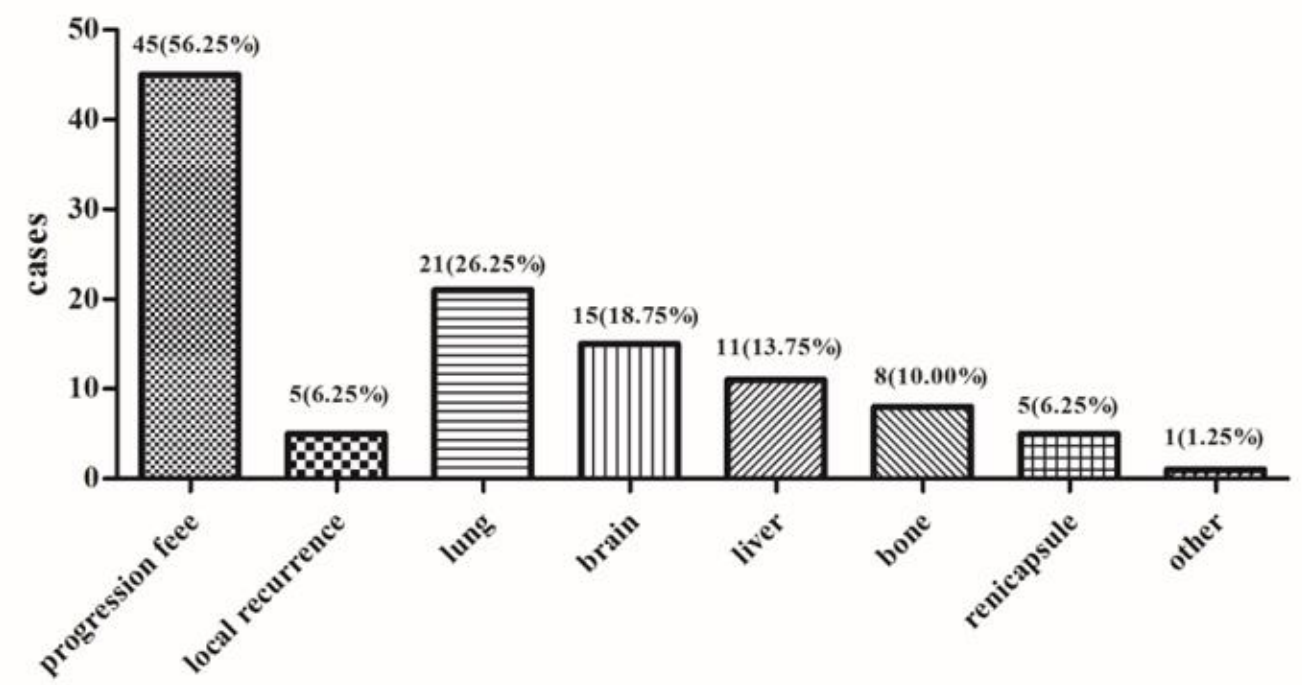

Figure 2. Recurrence and metastasis in postoperative NSCLC cases

\subsection{Risk factors for recurrence and progression of NSCLC}

Table 3 showed the results of recurrence-free survival of 80 NSCLC cases in the first, second, and third years after surgery. Cases with patients 65 years old and older were more prone to recurrence and progression compared with those under $65(\mathrm{P}=0.012)$. The more the differentiation, the lower the 3 -year recurrence-free survival rate $(\mathrm{P}=0.007)$. The later the postoperative $\mathrm{T}, \mathrm{N}$, and clinical stages, the lower the recurrence-free survival rate $(\mathrm{P}<0.001)$. The 3-year recurrence and progression rate of NSCLC patients with detected VM was $83.33 \%$ which was significantly higher than that of the no-VM group (20.00\%) (P $<0.001)$. Correlation analysis showed no significant correlation between VM and postoperative local recurrence of NSCLC $(r=0.120, P=0.289)$ but there was a significant correlation with distant metastasis $(\mathrm{r}=0.598, \mathrm{P}<0.001)($ Table 4).

Table 4. Associations of VM with NSCLC recurrence and metastasis

\begin{tabular}{cccc}
\hline Results of recurrence and progression $(\mathrm{n}=35)$ & $\mathrm{VM}+(\mathrm{n}=30)$ & Correlation coefficient & $\mathrm{P}$ value \\
\hline Local recurrence $(\mathrm{n}=5)$ & 3 & 0.120 & 0.289 \\
Distant metastasis $(\mathrm{n}=30)$ & 22 & 0.598 & $<0.001$ \\
Intrapulmonary $(\mathrm{n}=21)$ & 15 & 0.115 & 0.308 \\
Brain $(\mathrm{n}=15)$ & 13 & 0.488 & $<0.001$ \\
Bone $(\mathrm{n}=8)$ & 6 & 0.258 & $<0.05$ \\
Adrenal $(\mathrm{n}=5)$ & 5 & 0.333 & $<0.01$ \\
Liver $(\mathrm{n}=11)$ & 8 & 0.291 & $<0.01$ \\
Skin of left shoulder $(\mathrm{n}=1)$ & 1 & 0.145 & 0.199 \\
\hline
\end{tabular}

COX analysis revealed that histopathological type $(\mathrm{HR}=4.62,95 \% \mathrm{CI} 1.75-12.15, \mathrm{P}=0.002)$, tumor differentiation $(\mathrm{HR}=1.97,95 \% \mathrm{CI} 1.02-4.93, \mathrm{P}=0.003)$, $\mathrm{N}$ stage $(\mathrm{HR}=3.20,95 \% \mathrm{CI} 1.64-9.85, \mathrm{P}=0.004)$, T stage $(\mathrm{HR}=2.99,95 \% \mathrm{CI} 1.09-8.28, \mathrm{P}=0.034)$, clinical stage $(\mathrm{HR}=3.70,95 \% \mathrm{CI} 1.85-10-10.53, \mathrm{P}=$ $0.002)$, and $\mathrm{VM}(\mathrm{HR}=2.38,95 \% \mathrm{CI} 1.16-7.20, \mathrm{P}=0.002)$ were independent risk factors for postoperative recurrence and progression of NSCLC (Figure 3). 


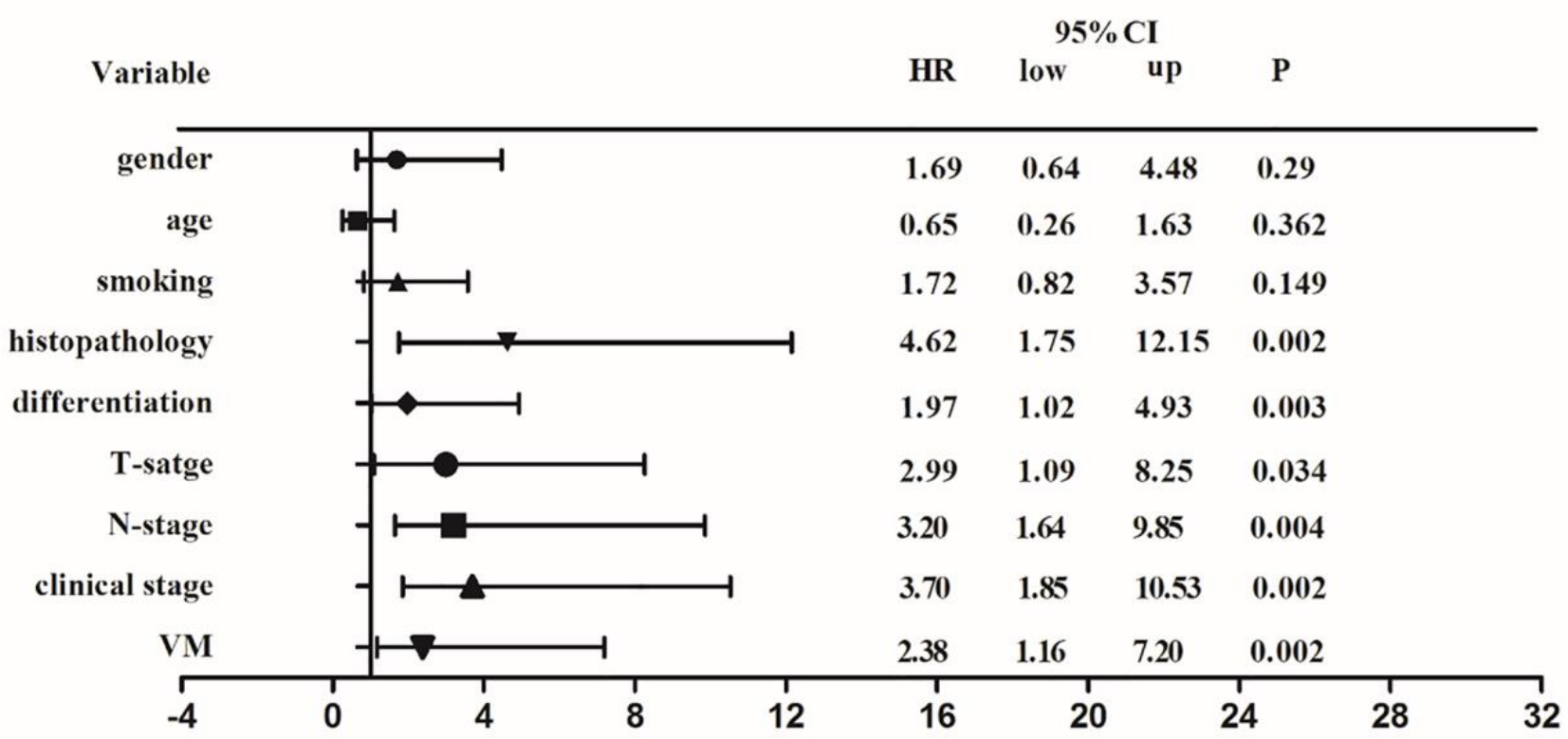

Figure 3. COX model for risk factors in postoperative NSCLC

\subsection{Effect of VM on postoperative RFS of NSCLC}

Among the 80 NSCLC cases, the average 3-year postoperative RFS was 32 months in those without VM and 18 months in the VM group with a significant difference $(\mathrm{P}<0.001)$ as shown in Figure 4A. Among the squamous carcinoma subgroup, the average 3-year RFS of VM cases was 18.9 months which was significantly shorter than that of non-VM cases $(34.4$ months, $\mathrm{P}<0.001$; Figure $4 \mathbf{B}$ ). Among the adenocarcinoma subgroup $(n=43)$, the average RFS of VM cases was 17.5 months which was significantly reduced compared to that of non-VM cases (29.7 months, $\mathrm{P}<0.001$; Figure $4 \mathrm{C}$ ). In the whole cohort, the average postoperative RFS of patients with adenocarcinoma was 24.6 months while that of the squamous carcinoma cases was 29.4 months. There was a significant difference in postoperative RFS between the two pathological types of NSCLC $(\mathrm{P}=0.028)$. In the VM-positive subgroup, the average RFS of patients with adenocarcinoma was 17.5 months and 18.9 months in patients with squamous carcinoma. There was no significant difference between the two groups $(P=0.620$; Figure 4D and Figure 4E). 
A

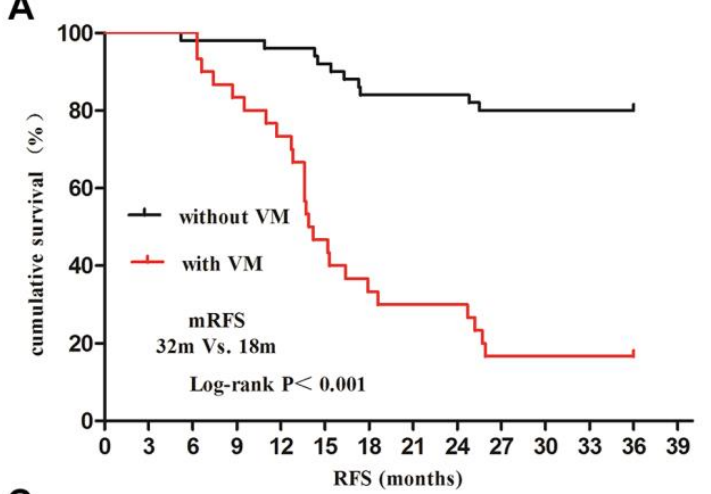

C

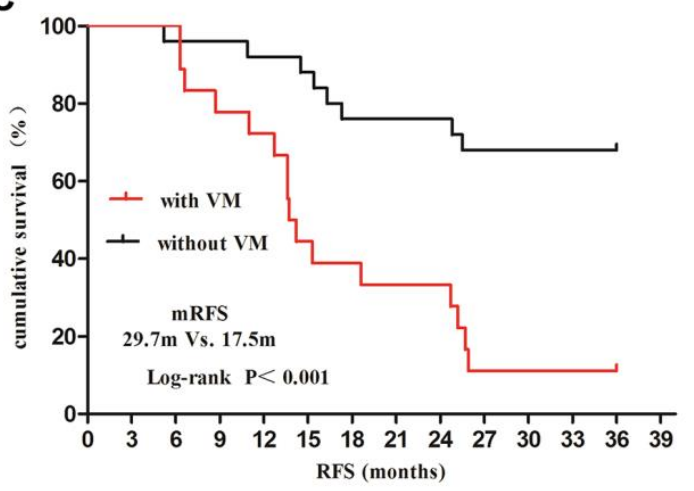

E

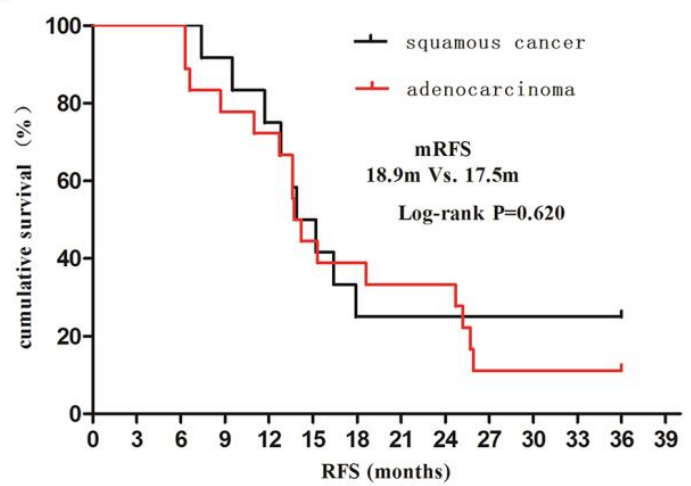

B

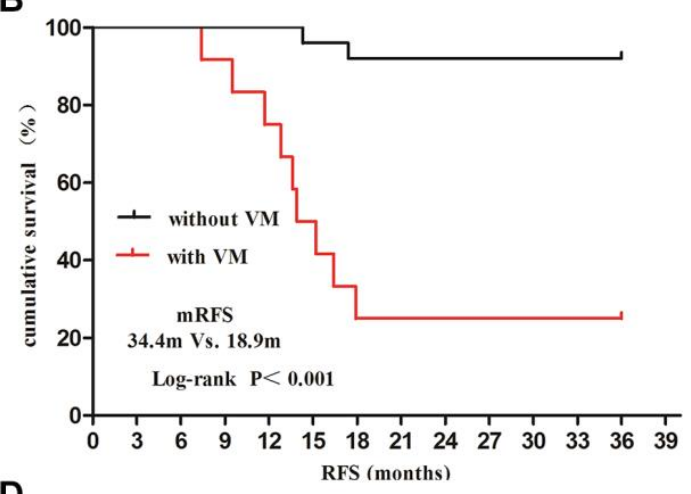

D

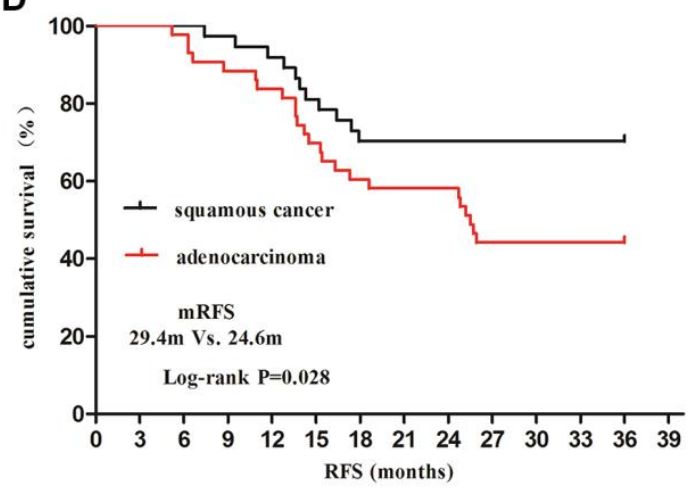

Figure 4. Presence of VM indicates poor postoperative survival. A: RFS of patients with and without VM in the whole population; B: RFS of patients with and without VM in the squamous carcinoma subpopulation; C: RFS of patients with and without VM in the adenocarcinoma subpopulation; D: RFS of patients with adenocarcinoma or squamous carcinoma without VM; E: RFS of patients with adenocarcinoma or squamous carcinoma with VM

\section{Discussion}

The above results showed that VM was detected in $37.5 \%$ postoperative NSCLC specimens and significantly correlated with postoperative NSCLC distant metastasis $(r=0.598, \mathrm{P}<0.001)$. VM in NSCLC increased the risk of postoperative recurrence and progression $(\mathrm{HR}=2.38,95 \% \mathrm{CI} 1.16-7.20, \mathrm{P}=0.002)$ and shortened the postoperative RFS (18 months versus 32 months, $\mathrm{P}<0.001)$. These results validated the value of VM in the risk assessment of postoperative NCSLC progression. Further related mechanistic studies are expected to provide references for subsequent clinical intervention strategies.

Local recurrence and distant metastasis usually occur within 1-3 years of surgery with most cases developing in the second year after surgery ${ }^{[7]}$. In this study, the postoperative 3-year recurrence and progression rate of NSCLC was $43.75 \%$, with $12.50 \%, 23.75 \%$, and $7.50 \%$ in the first, second, and third years, respectively which supported a retrospective analysis ${ }^{[2]}$. In terms of risk factors for recurrence and 
progression, studies had reported on the differentiation degree, pathological type, TNM stage, and clinical stage of tumors ${ }^{[8]}$. The current results suggested that the grade of tumor differentiation $(\mathrm{HR}=1.97)$, stage $(\mathrm{HR}=3.70)$, and pathological type $(\mathrm{HR}=4.62)$ were independent risk factors for postoperative recurrence and progression, consistent with $\mathrm{Xi}$ et al ${ }^{[7]}$. Many studies claimed that the older the patients are, the poorer the prognosis although age does not independently affect prognosis ${ }^{[9-11]}$. Univariate analysis in this study showed that age more than 65 years increases the 3-year recurrence and progression rate $(\mathrm{RR}=2.277, \mathrm{P}=$ 0.012) but COX model did not confirm it as an independent risk factor for prognosis $(\mathrm{HR}=1.69, \mathrm{P}=0.29)$. This may be related to the decline of organ reserve function and the increased risk of associated underlying diseases with older age but not to the malignant biological behavior of the tumor itself ${ }^{[12]}$.

In this study, VM could independently predict postoperative NSCLC progression $(\mathrm{HR}=2.38,95 \% \mathrm{CI}$ 1.16-7.20, $\mathrm{P}=0.002$ ). Other studies of solid tumors also suggested that VM increases the risk of postoperative recurrence and progression. In gastrointestinal tumors, VM results in a reduction of diseasefree survival $(\mathrm{DFS})(\mathrm{HR}=2.60,95 \% \mathrm{CI} 2.07-3.27, \mathrm{P}<0.001)^{[4]}$. In breast cancer, VM significantly decreases the postoperative DFS $(\mathrm{HR}=1.63,95 \% \mathrm{CI} 1.15-2.32, \mathrm{P}=0.002)^{[13]}$. Another study suggested that VM could independently affect the risk of postoperative NSCLC progression (HR $=3.39,95 \%$ CI 2.09$5.50, \mathrm{P}=0.002)^{[14]}$. The formation of $\mathrm{VM}$ involves many factors including stem-like cell differentiation, phenotypic transformation of tumor cells, changes of the tumor microenvironment, and tumor matrix remodeling which also constitute the relevant mechanisms of tumor resistance to postoperative adjuvant chemoradiotherapy ${ }^{[15]}$. Therefore, suppressing VM has become a major problem in clinical oncology.

The formation of VM results from further deterioration of tumor cell phenotype ${ }^{[16]}$. In this study, it was found that VM is related to the differentiation degree of tumor cells $(r=0.365, \mathrm{P}=0.001)$ which is consistent with most current research findings ${ }^{[17]}$. In this study, RFS in lung adenocarcinoma was significantly shorter than that of the lung squamous carcinoma ( 24.6 months versus 29.4 months, $\mathrm{P}=0.028$ ) while no significant difference was found in RFS between adenocarcinoma and squamous carcinoma in the VM positive subgroup (17.5 months versus 18.9 months, $\mathrm{P}=0.620$ ). These results suggested that VM may increase the malignant phenotype of squamous carcinoma and make it more prone to progression and metastasis. In addition, VM provides a structural basis for the direct contact of tumor cells with circulating blood which makes it easier for the hematogenous spread of tumor cells. As shown above, VM was not significantly correlated with local recurrence of NSCLC after surgery $(r=0.120, P=0.289)$ but it was significantly associated with distant metastasis $(\mathrm{r}=0.598, \mathrm{P}<0.001)$, suggesting that VM might be associated with hematogenous metastasis of tumors. Circulating tumor cells (CTCs) are strong evidence for distant metastasis of tumors. Stuart C et al. noted that CTCs found in the blood of patients with small cell lung cancer have the phenotype of VM-forming tumor cells in the primary lung lesion and are more likely to form $\mathrm{VM}^{[18]}$. Therefore, the increased risk of postoperative distant metastasis of NSCLC by VM may be related to elevated amounts of CTCs although this specific mechanism requires further investigation.

Postoperative standard adjuvant therapy for stage II-III NSCLC has certain progression-free survival (PFS) and overall survival (OS) benefits ${ }^{[19]}$. In this study, $32.5 \%$ of the patients were stage I cases. This study did not discuss whether patients with VM in this subgroup required adjuvant therapy and their reactions to the therapy were not assessed. In addition, it was not analyzed whether the mode of postoperative adjuvant therapy and the specific scheme of stage II-III patients affected the predictive value of VM in the prognosis of NSCLC. These limitations may have impacted the current findings and they deserve further investigations in follow-up studies. Furthermore, the distribution of HR in the COX model was too wide indicating that follow-up research would require further expansion of the sample size. 


\section{Conclusion}

This study confirmed that VM represents an independent risk factor for postoperative recurrence and progression of NSCLC in which it is expected to become a potential target for postoperative adjuvant therapy. Follow-up basic and clinical studies should further explore the mechanisms by which VM affects the postoperative recurrence and progression of NSCLC.

\section{Funding}

This study was supported by grants from the Natural Science Foundation of Jiangsu Province of China (Number: BK20181232), Talent Project of Jiangsu Province of China (Number: BRA2020186), Talent Project of Taizhou city (Number: RCPY201833) and the Major Programs of Natural Science Foundation of Higher Education in Jiangsu Province (Number: 19KJA480003).

\section{Disclosure statement}

The authors declare that there is no conflict of interest.

\section{Author contributions}

Jue and Aiqin designed the study, Baoyong performed and analyzed the results. Aiqin and Baoyong contributed equally to this work. Zhisheng and Jijun performed computational analyses. Yanqing provided technical assistance. Weichun and Yayun conceived, designed, and supervised the study. All authors had edited or commented on the manuscript. All authors had read and approved the final manuscript.

\section{References}

[1] Barnett R, 2017, Lung Cancer. Lancet, 390(10098): 928.

[2] Dziedzic DA, Rudzinski P, Langfort R, et al., 2016, Risk Factors for Local and Distant Recurrence after Surgical Treatment in Patients with Non-Small-Cell Lung Cancer. Clinical Lung Cancer, 17(5): e157-67.

[3] Ge H, Luo H, 2018, Overview of Advances in Vasculogenic Mimicry - A Potential Target for Tumor Therapy. Cancer Management and Research, 10: 2429-37.

[4] Ren HY, Shen JX, Mao XM, et al., 2019, Correlation between Tumor Vasculogenic Mimicry and Poor Prognosis of Human Digestive Cancer Patients: A Systematic Review and Meta-Analysis. POR, 25(3): 849-58.

[5] Ettinger DS, Wood DE, Akerley W, et al., 2014, Non-Small Cell Lung Cancer, version 1. JNCCN, 12(12): 1738-61.

[6] Sun H, Zhang D, Yao Z, et al., 2017, Anti-Angiogenic Treatment Promotes Triple-Negative Breast Cancer Invasion Via Vasculogenic Mimicry. Cancer biology \& therapy, 18(4): 205-13.

[7] Xi JJ, Yin JC, Wang L, et al., 2018, A Surveillance Method-Oriented Detection of Post-Operative Spatial-Temporal Recurrence for Non-Small Cell Lung Cancer. Journal of Thoracic Disease, 10(11): 6107-17.

[8] Lv C, An C, Feng Q, et al., 2015, A Retrospective Study of Stage I to IIIA Lung Adenocarcinoma After Resection: What is the Optimal Adjuvant Modality for Patients with an EGFR Mutation? Clinical Lung Cancer, 16(6): e173-81.

[9] Zhang Z, Liu D, Guo Y, et al., 2010, Effects of Multiple Factors on the Prognosis of pIIIa/N2 Patients 
with Non-Small Cell Lung Cancer. Chinese Journal of Lung Cancer, 13(8): 781-5.

[10] Komatsu H, Izumi N, Tsukioka T, et al., 2019, Prognosis Associated with Synchronous or Metachronous Multiple Primary Malignancies in Patients with Completely Resected Non-Small Cell Lung Cancer. Surgery Today, 49(4): 343-9.

[11] Yang H, Li X, Shi J, et al., 2018, A Nomogram to Predict Prognosis in Patients Undergoing Sublobar Resection for Stage IA Non-Small-Cell Lung Cancer. Cancer Management and Research, 10: 6611-26.

[12] Ucvet A, Yazgan S, Gursoy S, et al., 2018, Prognosis of Resected Non-small Cell Lung Cancer with Ipsilateral Pulmonary Metastasis. The Thoracic and Cardiovascular Surgeon.

[13] Shen R, Wu T, Huang P, et al., 2019, The Clinicopathological Significance of Ubiquitin-Conjugating Enzyme E2C, Leucine-Rich Repeated-Containing G Protein-Coupled Receptor, WW DomainContaining Oxidoreductase, and Vasculogenic Mimicry in Invasive Breast Carcinoma. Medicine, 98(16): e15232.

[14] Wang Y, Yang R, Wang X, et al., 2018, Evaluation of the Correlation of Vasculogenic Mimicry, Notch4, DLL4, and KAI1/CD82 in the Prediction of Metastasis and Prognosis in Non-Small Cell Lung Cancer. Medicine, 97(52): e13817.

[15] Liu Y, Mei L, Yu Q, et al., 2015, Multifunctional Tandem Peptide Modified Paclitaxel-Loaded Liposomes for the Treatment of Vasculogenic Mimicry and Cancer Stem Cells in Malignant Glioma. ACS Applied Materials \& Interfaces, 7(30): 16792-801.

[16] Quiros-Gonzalez I, Tomaszewski MR, Aitken SJ, et al., 2018, Optoacoustics Delineates Murine Breast Cancer Models Displaying Angiogenesis and Vascular Mimicry. British Journal of Cancer, 118(8): 1098-106.

[17] Wagenblast E, Soto M, Gutierrez-Angel S, et al., 2015, A Model of Breast Cancer Heterogeneity Reveals Vascular Mimicry as a Driver of Metastasis. Nature, 520(7547): 358-62.

[18] Williamson SC, Metcalf RL, Trapani F, et al., 2016, Vasculogenic Mimicry in Small Cell Lung Cancer. Nature Communications, 7: 13322.

[19] Bradbury P, Sivajohanathan D, Chan A, et al., 2017, Postoperative Adjuvant Systemic Therapy in Completely Resected Non-Small-Cell Lung Cancer: A Systematic Review. Clinical Lung Cancer, 18(3): 259-73, 258. 